\title{
A NOTE ON FREE GROUPS
}

\author{
R. G. BURNS
}

The object of this note is to point out a theorem of M. Hall, Jr. (Theorem 1), proved, but formulated in a weaker form, as Theorem 5.1 of [2]. We then show that results of Karrass and Solitar [5], and Howson [4], follow as relatively easy corollaries of this stronger statement (Corollaries 2 and 3 ).

Since the terminology is not fixed, we note for definiteness that by a right transversal for a subgroup $H$ in a group $G$ we shall mean a complete set of representatives of cosets $H g, g \in G$. Other terms used are defined in [1] and [6].

The strengthened theorem is as follows.

TheOREM 1. Let $F$ be any free group, $H$ any finitely generated subgroup and $a_{1}, \cdots, a_{k}$ any finite number $k$ of elements of $F$ not in $H$. Then there exists a subgroup $G$ of finite index in $F$ such that $G$ avoids the set $\left\{a_{1}, \cdots, a_{k}\right\}$ and contains $H$ as a free factor.

We sketch Hall's proof, modified slightly, with his reference to results of [3] replaced by a reference to the following converse of Schreier's theorem on subgroups of free groups.

Lemma 1. Let $F$ be a free group on a set $S$ of free generators and let $T$ be any right Schreier system in terms of $S$ (i.e. a set closed under taking left initial segments, including the identity 1). If there exists a function $\phi: T S \rightarrow T$ such that

$$
\phi(t s)=t s \quad \text { if } t s \in T \quad(t \in T, s \in S),
$$

and the mapping $\pi(s): t \rightarrow \phi(t s)$ is a permutation of $T$ for each $s \in S$, then those elements $t s \phi(t s)^{-1}(t \in T, s \in S)$ which are nontrivial, are distinct and form a set of free generators of a subgroup which has $T$ as a. right Schreier transversal.

This is merely a combination of Theorems 7.2.3 and 7.2.6 of [1].

Proof of the Theorem. Let $T_{1}$ be a right Schreier transversal for $H$ in $F$ and $\phi_{1}: F \rightarrow T_{1}$ the function associating with each element of $F$ its representative in $T_{1}$. By Schreier's theorem, $H$ is freely generated by those elements

$$
t_{1} s \phi_{1}\left(t_{1} s\right)^{-1} \quad\left(t_{1} \in T_{1}, s \in S\right)
$$

Received by the editors January 13, 1969. 
which are nontrivial. Let $T_{2} \subseteq T_{1}$ be the set of all those elements $t_{1}$, $\phi_{1}\left(t_{1} s\right)$ of $T_{1}$ such that $t_{1} s \phi_{1}\left(t_{1} s\right)^{-1} \neq 1$, together with 1 and the representatives in $T_{1}$ of the cosets $H a_{1}, \cdots, H a_{k}$. Since $H$ is finitely generated and $k$ is finite, it follows that $T_{2}$ is finite. Let $T$ be the result of adjoining to $T_{2}$ all (left) initial segments of elements of $T_{2}$. Clearly $T$ is finite.

We now define a function $\phi: T S \rightarrow T$. Whenever $\phi_{1}(t s) \in T(t \in T$, $s \in S)$, set $\phi(t s)=\phi_{1}(t s)$. This ensures that $\phi$ satisfies (1). Also, since the mapping $\pi_{1}(s): t \rightarrow \phi_{1}(t s)(t \in T)$ is certainly $(1,1)$, it follows that the partial function $\pi^{\prime}(s)$ from $T$ to $T$ which maps $t$ to $\phi_{1}(t s)$ whenever $\phi_{1}(t s) \in T$, can be extended to a permutation of $T$. For each $s$ take an arbitrary permutation $\pi(s): T \rightarrow T$, containing $\pi^{\prime}(s)$. It is easy to check that the equation $\phi(t s)=t \pi(s)$ then defines $\phi$ uniquely at every element of $T S$.

The mapping $\phi$ has been constructed to satisfy the conditions of Lemma 1. Therefore the elements

$$
1 \neq t s \phi(t s)^{-1} \quad(t \in T, s \in S)
$$

freely generate a subgroup $G$ with right Schreier transversal $T$ (and therefore $G$ has finite index). Among the expressions (3) occur all the free generators (2) of $H$. Hence $H$ is a free factor of $G$.

Finally we show that $a_{i} \notin G$ for $i=1, \cdots, k$. For each $a_{i}$ there is a nontrivial $t_{i} \in T$ such that $a_{i} t_{i}^{-1} \in H$, and hence $a$ fortiori such that $a_{i} t_{i}^{-1} \in G$. If $a_{i} \in G$ then $t_{i} \in G$. But $t_{i}$ is a nontrivial member of a Schreier transversal for $G$ in $F$ and therefore $t_{i} \notin G$. Thus $a_{i} \notin G$ and the proof is complete.

We have the following corollaries.

COROLlaRY 1. If $H$ is a finitely generated subgroup of a free group $F$, then $H$ is a free factor of some subgroup of finite index in $F$.

Corollary 2 (Karrass and Solitar [5]). If in a free group $F, H$ is a subgroup of infinite index containing a nontrivial normal subgroup of $F$, then $H$ has infinite rank.

Proof. Denote by $N$ the nontrivial normal subgroup of $F$, contained in $H$. Suppose $H$ has finite rank. Then by Corollary 1 it is a free factor of a subgroup $G$ of finite index. Since $H$ has infinite index, $H$ is a proper free factor of $G$. Thus $N$ is normal in $G$ and contained in a proper free factor of $G$. This is clearly impossible.

Corollary 3 (Howson [4]). If $A$ and $B$ are finitely generated subgroups of a free group $F$, then $A \cap B$ is finitely generated. 
For the proof we need the following lemma (see [6, p. 117, Exercise $32])$.

LEMMA 2. If $K_{1}$ is a free factor of a free group $K$ and $G$ is any subgroup of $K$, then $K_{1} \cap G$ is a free factor of $G$.

Proof of Corollary 3. We may assume $F$ has finite rank (if not replace $F$ by $\operatorname{sgp}\{A, B\})$. By Corollary 1 there exist subgroups $A * A_{1}$ and $B * B_{1}$ having finite indices in $F$ (and therefore also finite ranks). Applying Lemma 2 with $A * A_{1}, A$ and $\left(A * A_{1}\right) \cap\left(B * B_{1}\right)$ replacing $K, K_{1}$ and $G$ respectively, we deduce that $A \cap\left(A * A_{1}\right)$ $\cap\left(B * B_{1}\right),=A \cap\left(B * B_{1}\right)$, is a free factor of $\left(A * A_{1}\right) \cap\left(B * B_{1}\right)$. A second application, with $B * B_{1}, B$ and $A \cap\left(B * B_{1}\right)$ replacing $K, K_{1}$ and $G$ respectively, yields that $A \cap B$ is a free factor of $A \cap B * B_{1}$ and hence of $\left(A * A_{1}\right) \cap\left(B * B_{1}\right)$. But the latter group is of finite rank (since it has finite index in $F$ ) and hence so is $A \cap B$. This completes the proof.

Remarks. 1. Suppose in the above that rank $A=m$ and rank $B$ $=n$. Note that in the preceding proof $A \cap\left(B * B_{1}\right)$ has finite index ( $i$, say) in $A$. Therefore by Schreier's formula,

$$
\text { rank } A \cap\left(B * B_{1}\right)=i(m-1)+1 \text {. }
$$

Thus this certainly also provides a bound for rank $A \cap B$. However, Howson [4] and Hanna Neumann [7], [8] obtained the bound $2(m-1)(n-1)+1$ for rank $A \cap B$. I have been unable to deduce from the preceding a bound for rank $A \cap B$ in terms of $m$ and $n$, without using Howson's ideas.

2. Howson [4] remarked that if $A$ and $B$ both have finite index in $F$ then it follows (by Schreier's formula) that

$$
\text { rank } A \cap B \leqq(m-1)(n-1)+1 \text {. }
$$

This is also true when only one of $A$ and $B$ has finite index in $F$. For suppose without loss of generality that $B$ has finite index ( $j$, say). The group $F$ necessarily has finite rank ( $r$, say). We may assume that $r \geqq 2$ since the case $r=1$ is trivial. By Schreier's formula $n=j(r-1)+1$, whence $n \geqq j+1$. The index $i$ of $A \cap B$ in $A$ is the number of cosets of $B$ containing elements of $A$ and hence $i \leqq j \leqq n-1$. Thus by Schreier's formula, applied to $A \cap B$ in $A$,

$$
\text { rank } A \cap B=i(m-1)+1 \leqq(n-1)(m-1)+1 \text {. }
$$

\section{REFERENCES}

1. M. Hall, Jr., The theory of groups, Macmillan, New York, 1959.

2. - Coset representations in free groups, Trans. Amer. Math. Soc. 67 (1949), 421-432. 
3. M. Hall, Jr. and T. Rado, On Schreier systems in free groups, Trans. Amer. Math. Soc. 64 (1948), 386-408.

4. A. G. Howson, On the intersection of finitely generated free groups, J. London Math. Soc. 29 (1954), 428-434.

5. A. Karrass and D. Solitar, Note on a theorem of Schreier, Proc. Amer. Math. Soc. 8 (1957), 696-697.

6. W. Magnus, A. Karrass and D. Solitar, Combinatorial group theory, Intersciience, New York, 1966.

7. Hanna Neumann, On the intersection of finitely generated free groups, Publ. Math. Debrecen 4 (1956), 186-189.

8. - On the intersection of finitely generated free groups: Addendum, Publ. Math. Debrecen 5 (1957/58), 128.

Australian National University and

MCGILl UNIVERSITY 